\title{
Original
}

\section{Tratamiento actual de las masas renales pequeñas}

\author{
José I. Iriarte Soldevilla, Nerea Senarriaga Ruiz de la Illa, Isabel Lacasa Viscasillas, \\ Ana Loizaga Iriarte, Carmen Zubiaur Libano, Miguel Unda Urzaiz
}

Servicio de Urología. Hospital de Basurto. Bilbao, España

\section{Resumen}

Cada día son diagnosticados con más frecuencia de manera incidental Carcinomas Renales (CR), debido al aumento del uso de las técnicas de Radiodiagnóstico para el estudio de otras patologías. Su incidencia en los países desarrollados es superior al 40\%, siendo malignos el $80 \%$ de los tumores sólidos de riñón menores de $4 \mathrm{~cm}$. El tratamiento estándar de este tipo de tumores es la nefrectomía parcial con un índice de recidivas del 1-2\%. El mayor incremento en el diagnostico de esta patología se ha producido en pacientes entre los 70 y 90 años, grupo de edad que se acompaña de gran numero de comorbilidades. En las dos ultimas décadas y acompañando al desarrollo de las técnicas radiológicas, se han establecido entre nosotros dos técnicas, ablativas, que a través del frío, la Crioterapia (CA), y a través del calor, la Radiofrecuencia (RFA), consiguen la necrosis del tumor. Los resultados oncológicos a corto y medio plazo alcanzan un 95\% de remisiones en tumores menores de $4 \mathrm{~cm}$. y al ser técnicas que se pueden realizar por vía percutánea, tanto las complicaciones como las estancias hospitalarias han disminuido. Ya en 1995 Bosniak basándose en la experiencia de la observación del crecimiento y conducta de los CR pequeños durante más de 8 años propugna la postura de "Esperar y Ver" o "Vigilancia Activa”(VA). Presentamos la CA, nuestra serie de RAF y revisamos la literatura de la VA.

Conclusiones: En pacientes con edad avanzada, enfermedades concomitantes que desaconsejen la cirugía, monorrenos, con tumores renales múltiples o pacientes que rechacen la cirugía, las técnicas ablativas pueden ser utilizadas de manera segura y eficaz en manos expertas, alcanzando a medio plazo resultados oncológicos similares a la nefrectomía parcial, del mismo modo que en pacientes correctamente informados la vigilancia activa se ha demostrado como segura a medio plazo. Para mejorar las indicaciones, serán necesarias nuevas técnicas diagnósticas, que nos ayuden a diferenciar los tumores potencialmente más agresivos de los que no se van a desarrollar y series mayores y con más años de evolución para confirmar los resultados actuales.

Palabras clave: Carcinoma Renal. Crioterapia. Radiofrecuencia. Vigilancia Activa.

\section{Current treatment of small renal masses \\ Abstract}

Incidental diagnosis of renal carcinoma $(\mathrm{RC})$ is increasingly common due to widespread use of radiodiagnostic techniques for other conditions. In developed countries, incidental tumor account for more than $40 \%$ of detected tumors, and $80 \%$ of solid kidney tumors less than $4 \mathrm{~cm}$ in size are malignant. Standard treatment for these tumors is partial nephrectomy, and their relapse rate is $1 \%-2 \%$ The higher increase in diagnosis of this disease has occurred in patients aged 70 to 90 years, a group where associated comorbidities are very common. In the past two decades, in parallel to development of radiographic techniques, two ablation procedures achieving tumor necrosis through cold, cryotherapy, and through heat, radiofrequency, have become established. These procedures achieve 95\% short- and long-term remissions in tumors less than $4 \mathrm{~cm}$ in size. In addition, since these procedures may be performed percutaneously, both complications and hospital stay have decreased. As early as in 1995, Bosniak, based on observation of the growth and behavior of small RCs for longer than 8 years, advocated a watchful waiting or active surveillance attitude. This article reports cryotherapy, our radiofrequency series, and a literature review.

Conclusions: In the event of elderly patients, concomitant diseases advising against surgery, multiple renal tumors, a solitary kidney, or patients who reject surgery, ablation procedures may be safe and effective when performed by expert hands, achieving mid-term oncological results similar to partial nephrectomy. Active surveillance has also been shown to be safe in the mid-term in adequately informed patients. To improve indications, new diagnostic procedures that help us differentiate the potentially more aggressive tumors will be required. Larger series and longer follow-ups are neede to confirm current results.

Keywords: Renal carcinoma. Cryotherapy. Radiofrequency. Active surveillance. 
C ada año son diagnosticados más de 200.000 casos de Carcinoma Renal y se producen más de 100.000 muertes a nivel mundial atribuidas a esta enfermedad, con mayor incidencia en Norte América, Europa y Australia ${ }^{1}$. La detección de Tumores Renales continua creciendo, debido al aumento de la utilización de las técnicas radiodiagnósticas, (Ecografía, TAC, RMN) (Fig. 1) en el estudio de otras patologías. Estos tumores detectados incidentalmente, tienden a ser más pequeños, presentar estadíos y grados histológicos más bajos y asociarse con una menor incidencia de metástasis y una supervivencia mayor. En los países desarrollados la detección incidental de Tumores renales es superior al 40\% . En el 80\% de los casos los tumores sólidos de riñón menores de $4 \mathrm{~cm}$ son malig$\operatorname{nos}^{2,3, \mathrm{C} 18}$, (Fig. 1). Está demostrada la eficacia oncológica de la cirugía conservadora, equivalente a la radical, con un 1 a $2 \%$ de recidivas ${ }^{4}$.

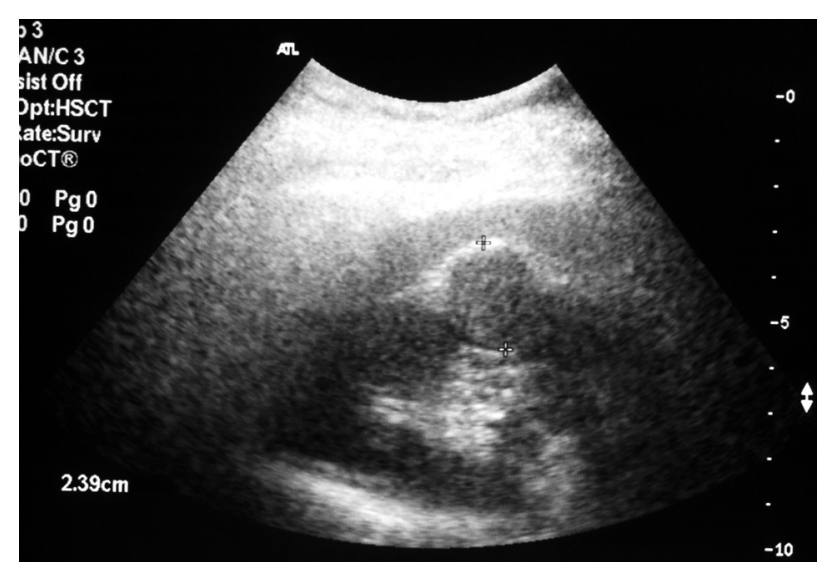

FIGURA 1. Masa Renal incidental menor de $4 \mathrm{~cm}$.

Se define como cirugía mínimamente invasiva al conjunto de técnicas diagnósticas y terapéuticas que por visión directa o endoscópica, o por otras técnicas de imagen, utilizan las vías naturales o mínimos abordajes para introducir herramientas y actuar en diferentes partes del cuerpo. Sus ventajas, la disminución de la respuesta inflamatoria, la mejoría en la respuesta inmunológica, la disminución del dolor postoperatorio y el acortamiento del periodo de recuperación y la estancia hospitalaria. Como técnicas mínimamente invasivas deben cumplir los criterios de conservación de parénquima, baja morbilidad, control oncológico similar a otras técnicas, beneficios demostrables en cuanto a calidad de vida y disminución de costos. En Europa se estima que en el 2009 el 25\% del total de operaciones se llevarán a cabo mediante técnicas de cirugía mínimamente invasivas ${ }^{5}$.

En tumores menores de $4 \mathrm{~cm}$ el tratamiento standard es la Nefrectomía parcial, que proporciona tasas de supervivencia a 5 y 10 años superiores al 90\% (X1) siendo la vía laparoscópica la que se impone en estos momentos, comenzando a ponerse en duda si el límite de los $4 \mathrm{~cm}$ es válido ${ }^{6}$.

Vamos a revisar las técnicas mínimamente invasivas,Crioterapia y Radiofrecuencia, considerando como tal la vía percutánea por su menor morbilidad y estancia hospitalaria comparada con la laparocópica $^{7}$ y la Vigilancia Activa.

\section{CRIOTERAPIA}

La primera referencia al tratamiento de enfermedades mediante el frío aparece en el Edwin Smith Surgical Papyrus (bf 3000) en Egipto. La moderna Criocirugía se populariza en 1961 por un neurocirujano, Cooper en el tratamiento del Parkinson, pero no es hasta la década de los 80-90 con el perfeccionamiento de la ecografía, cuando la técnica evoluciona. Paralelamente al desarrollo de la criocirugía, Shulman (1965), Yantorno (1966) y Ablin (1968), comienzan a investigar, los efectos inmunológicos y demuestran la creación de anticuerpos como consecuencia de la congelación de los tejidos, naciendo con Tanaka (1982) el concepto de Crioinmunología ${ }^{8}$.

\section{Criobiologia ${ }^{9-19}$}

El frío produce una serie de lesiones tisulares, hasta producir la muerte celular por necrosis $\mathrm{y}$ apoptosis. Entre $-7 \mathrm{y}-10^{\circ} \mathrm{C}$ se produce la cristalización intracelular, que lleva a la deshidratación celular. Entre -15 y $-40^{\circ} \mathrm{C}$ aparece la congelación del liquido intracelular, cesando todos los procesos metabólicos, destruyéndose los organelos intracelulares y la membrana plasmática.

A nivel intravascular el hielo, provoca fisuras $y$ desgarros en los vasos, favoreciendo la formación de coágulos y la perdida de continuidad del endotelio, lo que provoca la formación de trombos y la consiguiente necrosis de los tejidos, por ellos irrigados. Puede haber células que sobrevivan al frío, pero se especula que temperaturas subletales, lleven a la célula a un proceso de muerte programada. La Apoptosis ocurre entre los $6 \mathrm{y}-10^{\circ} \mathrm{C}$ y el tiempo de activación es de unos minutos habiéndose demostrado en estudios 
"in vitro". Modelos experimentales demuestran que solo el $15 \%$ de las células del Carcinoma Renal, sobreviven a $-20^{\circ} \mathrm{C}$. Las variables determinantes en el proceso de congelación son:

A) Temperatura mínima alcanzada. A menor temperatura mayor deshidratación celular y aumento del hielo intracelular.

B) Velocidad de congelación. Siendo variable la velocidad necesaria para conseguir la congelación intracelular y por tanto la muerte celular, según el tipo de tejido. Siendo deseable la mayor velocidad posible.

C) Tiempo de temperatura deseada aumentando el periodo de congelación se puede producir el fenómeno de recristalización con lo que se produce un aumento del volumen y por lo tanto un aumento de las lesiones.

D) Número de ciclos y velocidad de calentamiento durante el deshielo lento, se modifica el ambiente extracelular, cuando el hielo se hace liquido, las células que permanecen vivas se encuentran en un ambiente hipotónico, con lo que el agua entra a través de su membrana y provoca la rotura celular. En un segundo ciclo la resistencia del parénquima a la congelación es menor, consiguiendo su congelación a mayor velocidad.

E) Número de criosondas. En la punta de la criosonda la temperatura que se puede alcanzar es de $190^{\circ} \mathrm{C}$ y en la periferia de la bola de $0^{\circ} \mathrm{C}$. A mayor número de criosondas, mayor superficie a tratar a temperatura adecuada ${ }^{43}$.

\section{Técnica}

Consiste en la colocación de una ó varias criosondas a nivel del tumor, dependiendo de su tamaño. La introducción a través de ellas del gas Argón, consigue por el efecto Joule ${ }^{20}$ temperaturas inferiores a los $-40^{\circ} \mathrm{C}$. Cada uno de los ciclos de enfriamiento, que suelen ser 2 de unos 10 min., se sigue de un ciclo de calentamiento, mediante el gas Helio, con el objetivo de optimizar la cantidad de tejido dañado. Para asegurar la temperatura adecuada a nivel de los márgenes, varios autores recomiendan, que la bola de hielo se extienda $1 \mathrm{~cm}$ más allá del margen tumoral ${ }^{21}$. En un comienzo se utilizo la vía abierta, que dejó paso a la vía laparoscópica, que ofrece las mismas posibilidades de movilización y exposición del riñón a la monitorización por ultrasonidos, con mucha menor morbilidad. La técnica percutánea ha demostrado la misma eficacia a corto plazo y menor morbilidad ${ }^{7,22}$. El tipo de anestesia, estará en relación con la técnica quirúrgica empleada. El control más frecuente es ecográfico, en el que se observa como un área hipoecoica engloba al tumor y mediante termosensores que monitorizan en tiempo real, nos aseguran que se han alcanzado las temperaturas adecuadas, durante el tiempo necesario.

El seguimiento se puede realizar mediante RMN o TAC por su carácter de test funcional, Tras la crioterapia se produce edema e inflamación como respuesta a la agresión y necrosis como respuesta al tratamiento, la ecografía tiene un valor limitado pues la masa aunque disminuye de tamaño, persiste en la mayoría de los $\operatorname{casos}^{23}$. En los días inmediatos al tratamiento se evidencia una lesión infartada de un tamaño mayor que la lesión primitiva con una mínima captación de contraste ${ }^{24}$. Posteriormente se produce una disminución gradual del diámetro de la lesión y en la mayoría de los casos a los tres meses se produce la resolución completa o la presencia de una lesión residual. El criterio decisivo en el control de la lesión es una captación de contraste menor de 10 ud. Hounsfield ${ }^{23}$. En ocasiones se evidencia durante los 6 primeros meses realces focales o en los bordes e infiltración de la grasa perirrenal, que desaparecen posteriormente ${ }^{25}$. Se ha demostrado, en pacientes a los que se les ha practicado nefrectomía parcial por persistencia de realces focales en el interior del tumor, pasados los 6 meses, que no se correspondian con persistencia de tejido tumoral ${ }^{26}$.

\section{Crioinmunología ${ }^{27-37}$}

Han sido demostradas evidencias de inmunidad especifica provocada por la crioablación de tumores, en varios estudios realizados en animales y en modelos in vitro. La elevación de los niveles de citoquinas inflamatorias y de interleuquina-6 tras la crioterapia, quizás pudieran darnos las bases para explicar la regresión de metástasis, comunicadas tras la crioablación del tumor primario en diferentes tejidos.

\section{Resultados}

Tras la crioablación tanto la Tensión arterial como la función renal no presentan cambios significativos, incluso en pacientes monorrenos ${ }^{38}$. Existen ya series con más de 5 años de evolución, con una supervivencia cáncer especifica del $95 \%{ }^{39,44-46}$ y 
una del Washington University de St.Luis con un 0\% de recurrencias con 3 años de seguimiento ${ }^{47}$. La mayoría de las complicaciones son menores, siendo la más frecuente el dolor a nivel de la inserción de las criosondas. Han sido descritas lesiones esplénicas y fracturas renales en casos de riñones frágiles o con antecedentes de pielonefritis ${ }^{40}$, incluso un caso de muerte ${ }^{41}$. Se están tratando tumores mayores de $4 \mathrm{~cm}$, viendo que es posible y seguro, si bien puede ser necesario el retratamiento por ablaciones incompletas. Los resultados oncológicos a corto plazo son buenos pero se desconoce la eficacia oncológica a largo plazo ${ }^{42}$.

\section{RADIOFRECUENCIA}

La RFA es una técnica ampliamente utilizada, cuya eficacia terapéutica radica en la posibilidad de lesionar los tejidos de manera controlada, por medio del calor. Se trata de una técnica segura pues no estimula el músculo ni los nervios y el calor se disipa en un área muy próxima a la interfase electrodotejido.

La historia de la RFA se inicia en 1891 en que D’Asorval, descubre que una corriente alterna $>10 \mathrm{KHz}$ produce calor sin contracción muscular. En 1900 Rivierte utiliza RFA para coagular y cauterizar tejidos. En 1908 Clusi y Obvie crean el primer prototipo de electrocirugía comercial. Entre 1980-89 se utiliza la RFA para el tratamiento de las arritmias cardiacas y en 1990 Rosi et al. y Mc Gihar et al. comienzan a tratar tumores sólidos de hígado.

La corriente alterna atrae y repele iones y la interferencia entre los iones oscilantes genera calor. La técnica se basa en la capacidad de las ondas de RF de generar el movimiento de iones en los tejidos adyacentes al extremo de una aguja conductora. La corriente reduce su densidad según se aleja del electrodo, sólo el área diana sufre una agitación suficiente para producirse una necrosis coagulativa (Tabla 1).

En Mayo del 2005 comenzamos a tratar en nuestro Hospital, tumores renales menores de $4 \mathrm{~cm}$, en pacientes monorrenos o con tumores múltiples y pacientes con patologías concomitantes que contraindiquen la cirugía mediante ablación con radiofrecuencia. Se han tratado cuatro tumores mayores de $4 \mathrm{~cm}$, uno por negarse el paciente a la cirugía y tres por alto riesgo quirúrgico. Se han realizado 66 RAF sobre 55 tumores en 52 pacientes, (35 hombres y 17 mujeres), con una edad media de 65 años (4781), 33 de Riñón derecho y 22 del izquierdo (Tabla 2).
Tabla 1. Efecto del calor sobre la célula

\begin{tabular}{ll}
\hline $42-45^{\circ} \mathrm{C}$ & $\begin{array}{l}\text { Células más susceptibles de ser } \\
\text { dañadas por quimio o radioterapia }\end{array}$ \\
$\begin{array}{l}70-80^{\circ} \mathrm{C} \\
\text { Citotoxicidad } \\
90^{\circ} \mathrm{C}\end{array}$ & $\begin{array}{l}\text { Desnaturalización de las proteínas } \\
\text { e inicio de la coagulación }\end{array}$ \\
Desecación celular & $\begin{array}{l}\text { La célula pierde su contenido en } \\
\text { agua }\end{array}$ \\
$100^{\circ} \mathrm{C}$ & La célula explota \\
Vaporización & \\
$200^{\circ} \mathrm{C}$ & Carbonización \\
\hline
\end{tabular}

Tabla 2. Características de la serie

\begin{tabular}{ll}
\hline Número de Pacientes & 52 \\
Numero de Tumores & 55 \\
Numero de Ablaciones & 66 \\
Diámetro Tumoral & $3,17(2-5,2)$ \\
Edad media & $65(47-81)$ \\
Sexo & 35 varones/17 mujeres \\
Lado & Derecho 33/Izquierdo 22 \\
\hline
\end{tabular}

El procedimiento se realiza bajo sedación, con control ecográfico, utilizándose un generador con una potencia de 200W (RF3000) y una Aguja electrodo (LeVeen), Figs. 2 y 3 . En todos ellos se practica en el mismo acto una biopsia: 41 Adenocarcinomas (82\%), 11 Oncocitomas, 1 Metástasis de Melanoma, 1 Angiomiolipoma, 1 Adenoma (Tabla 3).

En algunos tumores de cara anterior se introduce suero fisiológico para separar el paquete intestinal de la lesión y evitar complicaciones. En un tumor mayor de $4 \mathrm{~cm}$ se realizó la inyección de etanol a nivel tumoral, técnica ampliamente estudiada en diferentes modelos animales y en humanos a nivel de hígado, que produce un aumento del área de coagulación, sin incrementar las complicaciones ${ }^{48}$. Algunos autores realizan una embolización previa a RAF en tumores mayores de $3,5 \mathrm{~cm}^{49}$. A las $24 \mathrm{~h}$ se practica un control ecográfico con contraste y el paciente es dado de alta hospitalaria. Un mes después se practica TAC, a los 3 meses $\mathrm{RMN}^{50}$ (Figs. 4, 5 y 6), alternando estas dos técnicas durante los dos primeros años, pasando posteriormente al protocolo de tumores renales de nuestro Servicio.

El seguimiento medio ha sido de 15 meses (239). No hubo dificultades durante el procedimiento, salvo en un paciente monorreno con 3 tumores, en 


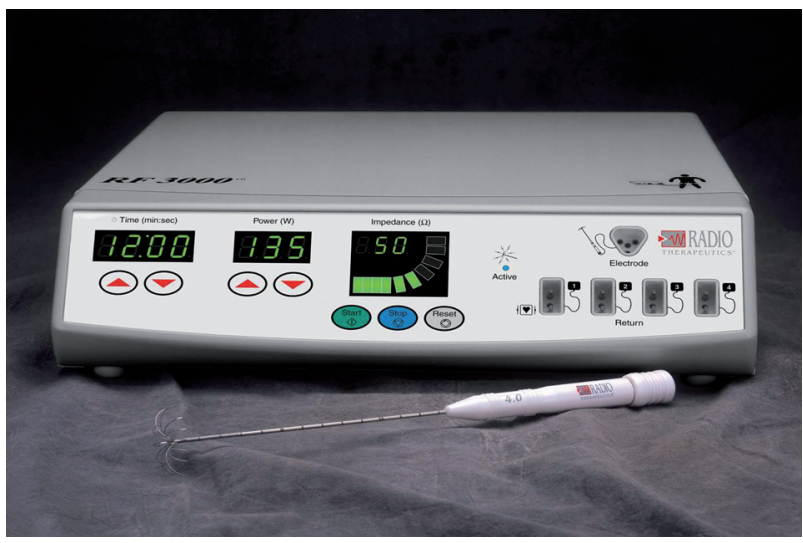

FIGURA 2. Generador y Aguja utilizados en la Radiofrecuencia.

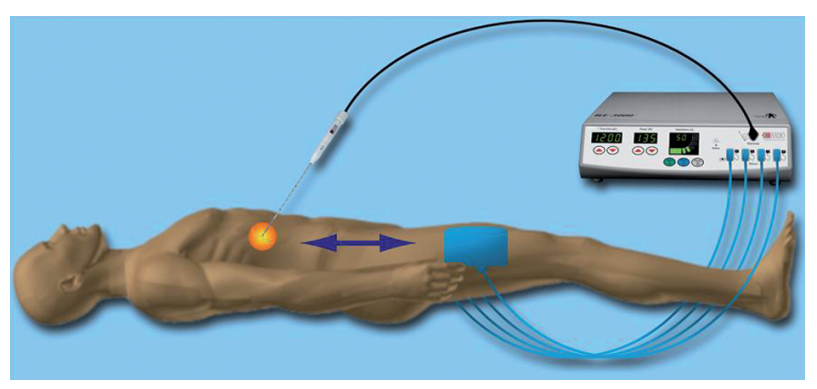

FIGURA 3. Circuito de Radiofrecuencia.

Tabla 3. Anatomía patológica de los tumores

\begin{tabular}{ll}
\hline Adenocarcinoma & $41(82 \%)$ \\
Oncocitoma & 11 \\
Adenoma & 1 \\
Angiomiolipoma & 1 \\
Metástasis de Melanoma & 1 \\
\hline
\end{tabular}

el que el vapor de agua que se forma durante la ablación, dificultó la visión ecográfica y obligó a dejar para una segunda sesión la ablación del tercer tumor y la aparición de una quemadura a nivel de la placa de retorno en un paciente con un tumor mayor de $5 \mathrm{~cm}$. En la ecografía de control se observan con frecuencia hematomas asintomáticos que no han requerido tratamiento. El 90\% de los pacientes, presentan dolores las primeras horas, que se pueden acompañar de nauseas y ceden con tratamiento habitual; este dolor puede deberse al hematoma o por lesión de nervios intercostales o lumbares afectando al dermatoma ${ }^{51}$. Únicamente un paciente requirió ingreso superior a $24 \mathrm{~h}$ por dolor. Un paciente ingresó a los 15 días por un cuadro febril con urocultivos y hemocultivos negativos que cedió con tratamiento antibiótico. Dos pacientes han fallecido por causas no relacionadas
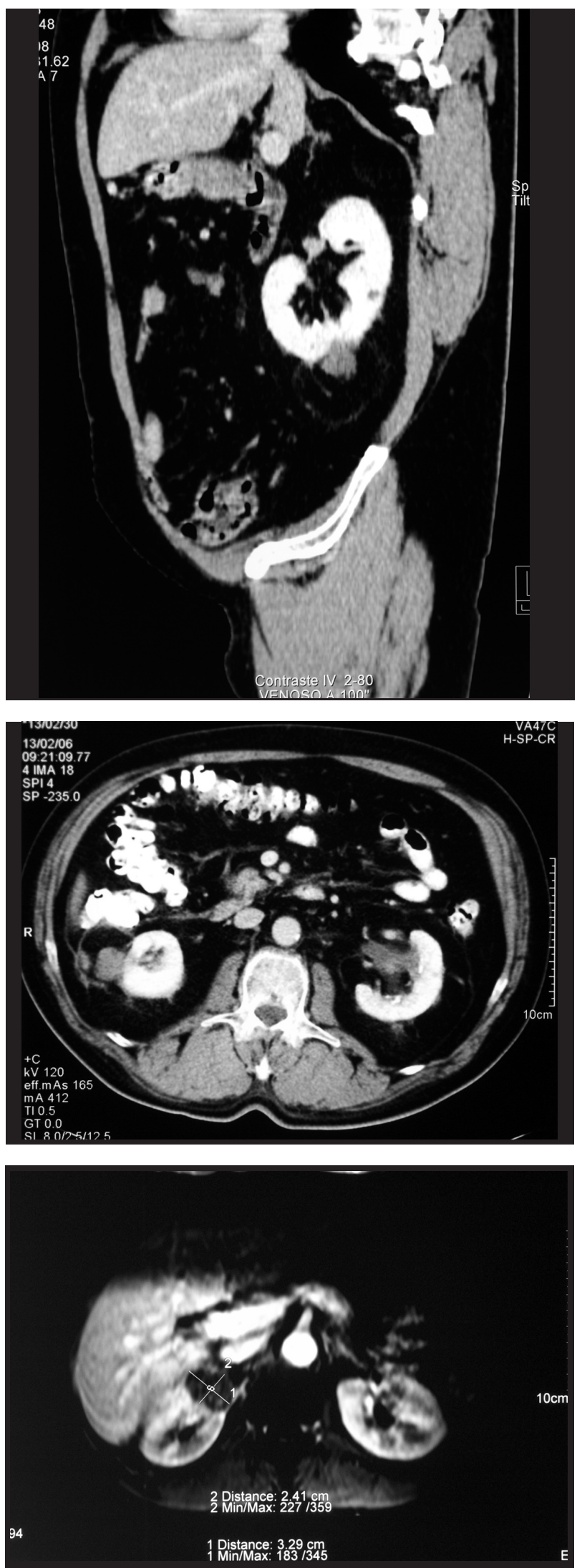

FIGURAS 4, 5 y 6. TAC y RMN de control con ausencia de captación de las masas y hematoma peritumoral. 
con el tratamiento, ni con su carcinoma renal ( IAM y hemorragia digestiva). Ha sido necesario repetir el tratamiento en 7 pacientes $(10,6 \%)$ : 4 por tratamiento incompleto y 3 por recidiva, confirmándose por estudio genético que dos de ellos padecen un Von Hippel Lindau (VHL). Un paciente fue rescatado por nefrectomía radical, sin dificultades sobreañadidas durante la intervención salvo la fibrosis en el trayecto de la aguja, otro paciente se encuentra con tratamiento sintomático por padecer un Carcinoma de próstata hormonorrefractario y el resto en remisión (93,9\%) (Tablas 4 y 5).

Tabla 4. Resultados

\begin{tabular}{ll}
\hline Seguimiento (meses) & $15(2-39)$ \\
Ingreso < 24 horas & $54(98,9 \%)$ \\
Ingreso > 24 horas & $1(1,1 \%)$ \\
Reingresos & 1 (fiebre) \\
Pacientes fallecidos & 2 ( IAM, hemorragia digestiva) \\
Reablaciones & $11(22 \%)$ \\
Nefrectomia salvataje & 1 \\
Tto. Sintomático & 1 (CAP Hormonorrefractario) \\
Remisiones & $93,9 \%$ \\
\hline
\end{tabular}

Tabla 5. Complicaciones

\begin{tabular}{ll}
\hline Dolor primeras horas & $48(74 \%)$ \\
Náuseas & $30(45 \%)$ \\
Hematoma & $36(59 \%)$ \\
Hematoma sintomático & $1(1,5 \%)$ \\
Fiebre & $1(1,5 \%)$ \\
Quemadura & $1(1,5 \%)$ \\
\hline
\end{tabular}

\section{Vigilancia activa}

El mayor incremento de incidencia del CR, se ha producido en pacientes de 70 a 90 años $^{52-54}$. A este grupo de edad le acompañan un gran número de comorbilidades. La presentación incidental del CR ha pasado del 10 al 60\%55, por todo ello, ya en 1995 Bosniak propugna esta conducta basado en la experiencia de la observación del crecimiento y la conducta de pequeñas masas diagnosticadas incidentalmente, con un seguimiento de hasta 8 años ${ }^{56}$.

La indicación incluiría pacientes que se niegan a ser intervenidos, que la intervención esté absolutamente contraindicada por comorbilidades severas o un riesgo quirúrgico inaceptable y las indicaciones relativas, pacientes con patologías malignas conco- mitantes o enfermedades de manejo médico difícil y pacientes a los que la extirpación quirúrgica supondría su entrada en diálisis ${ }^{52}$.

El limite que parece puede ser seguro, serían los $3,5 \mathrm{~cm}$ de diámetro ${ }^{57}$, si bien hay autores a los que les parece razonable y seguro en pacientes ancianos el seguimiento en tumores mayores de $5 \mathrm{~cm}^{58}$.

Parece que el crecimiento tumoral de las masas pequeñas es a menudo limitado y que el riesgo de metástasis sin crecimiento tumoral es mínimo ${ }^{52}$; sin embargo diferentes autores presentan una incidencia del 2 al 7\% de metástasis en CR pequeños, todos ellos sintomáticos. Se han publicado únicamente 4 progresiones metastásicas en pacientes con CR en vigilancia activa y únicamente uno de ellos fue asintomático en el momento de la progresión, de lo que se deduce que el riesgo metastásico es mínimo pero no carente de importancia55,56,59-61. La demora vigilada en el tratamiento de estas lesiones pequeñas no supone una complicación, no limita las múltiples opciones de tratamiento ni se corre un alto riesgo de progresión de la enfermedad ${ }^{62-64}$.

La biopsia percutánea con aguja de las masas renales es un método seguro con las nuevas técnicas y nos puede ayudar a seleccionar mejor los pacientes susceptibles de seguir la vigilancia activa, pero son necesarios nuevos biomarcadores, técnicas radiológicas, y descubrir características moleculares o genómicas de las biopsias con aguja que nos ayuden a diferenciar entre tumores indolentes o potencialmente agresivos, para poder indicar la vigilancia activa en pacientes jóvenes ${ }^{65-70}$.

\section{DISCUSIÓN}

Entre el 15 y el 22\% de las lesiones menores de $4 \mathrm{~cm}$ tratadas, son benignas y no es fácil en estos momentos el diagnostico en cuanto a la posible agresividad de la misma, previo al tratamiento. Además se conoce poco sobre la historia natural de estas lesiones. La Nefrectomía parcial se ha demostrado igual de efectiva que la radical, pero ambas presentan una alta morbilidad ${ }^{23}$.

En el año 2006 P. Whelan analiza las publicaciones aparecidas entre enero de 1999 y enero del 2006, concluyendo, que las técnicas ablativas no quirúrgicas representan un tratamiento alternativo para los $\mathrm{CR}<4 \mathrm{~cm}$ en pacientes seleccionados, debido al avance en las técnicas de imagen y la posibilidad de repetir estos tratamientos ${ }^{71}$. Ese mismo año la Asociación Americana de Urología en un Board of 
Directors del mes de Octubre, refiere que estas técnicas son apropiadas para el tratamiento de masas renales en pacientes seleccionados y que Urólogos entrenados en estas técnicas, pueden realizarlas de una manera efectiva y segura.

En el 2008 Kunkle revisa y analiza, 99 estudios con 6.471 lesiones y concluye, que las tres estrategias son viables para el tratamiento de los CR pequeños, basándose en los resultados a corto y medio plazo desde el punto de vista Oncológico ${ }^{72}$.

Para las Guías Clínicas Europeas del 2008, se trata de técnicas con un grado B de recomendación, para pacientes no adecuados para cirugía, con tumores periféricos y pequeños. Consideran una desventaja la falta de una adecuada evaluación histopatológica, que su eficacia debe ser mejor evaluada y que existe un nivel de evidencia 3 en cuanto a que posibilitan el tratamiento de pacientes con reducida condición física o aquellos en que no es posible la cirugía convencional.

En algunos foros, se puede escuchar el miedo de la comunidad urológica a perder esta patología en manos de los radiólogos intervencionistas, si se utiliza la vía percutánea en lugar de la laparoscópica. En este sentido, Liaron Long realizó una revisión de la literatura comparando la CA con la RFA, en la que concluye que los resultados son similares, sin embargo la RFA requiere más reablaciones, para conseguir el 95\% de supervivencia cáncer específica y que la reablación está directamente relacionada con la especialidad del cirujano; el $72 \%$ de las reablaciones son realizadas por radiólogos, a pesar de que sólo realizan el $31,4 \%$ de las primeras ablaciones, del mismo modo que es más frecuente la nefrectomía de salvataje tras la CA que tras la RFA porque el $89 \%$ de las $\mathrm{CA}$ son realizadas por Urólogos ${ }^{73}$.

Nuestra serie de RFA, presenta unas cifras similares, a las de la literatura, únicamente está aumentado el \% de reablaciones, que quizás se pueda explicar, por la utilización de la técnica en cuatro tumores mayores de $4 \mathrm{~cm}$ y contar con dos paciente que padecen un VHL. Otra curiosidad es no contar con anatomías patológicas no valorables, quizás también por no contar con tumores menores de 2 $\mathrm{cm}$.

La vigilancia activa no ha demostrado peores resultados, a corto y medio plazo respecto a la técnicas ablativas y a la nefrectomía parcial, quedando siempre la posibilidad del tratamiento ante cual- quier signo de progresión y por tanto su indicación puede depender únicamente, de la preferencia del paciente tras una adecuada información.

\section{CONCLUSIONES}

En paciente añosos, con enfermedades concomitantes que contraindiquen la cirugía, en pacientes monorrenos o con tumores múltiples y en aquellos pacientes que rechacen la cirugía, las técnicas ablativas, han demostrado unos resultados a corto y medio plazo similares a la nefrectomía parcial. La técnica percutánea se ha demostrado superior en cuanto a disminución de la morbilidad y estancia hospitalaria y en manos del urólogo los resultados son mejores respecto a otras especialidades. Del mismo modo en un paciente correctamente informado, la vigilancia activa, es otra posibilidad segura y eficaz a medio plazo. La aparición de nuevas pruebas diagnosticas, que nos permitan diferenciar la agresividad de los tumores, supondrá una ayuda, a la hora de indicar alguna de las nuevas alternativas terapéuticas. A día de hoy no se ha establecido si alguno de estos tratamientos altera la historia natural de las masa renales pequeñas y serán necesarias más series y años de evolución para confirmar los resultados actuales a largo plazo.

\section{REFERENCIAS}

1. Hegarty NJ, Kaouk JH, Spaliviero M, Desai MM, Novick AC, Remer E. Renal cryoablation: 5 year outcomes.AUA 2006 Abst (1091).J Urol. 2006: 175 (suppl): 351.

2. Aso Y, Homma Y. A survey on incidental renal cell carcinoma in Japan J Urol. 1992;147(6):1478-1481.

3. Siow WY, Yip SKH, Tan PH, Cheng WS, Foo KT. Incidental renal cell carcinoma: clinical and pathological staging. J R Coll Surg Edinb. 2000;45(5):291-295.

4. Fergany AF, Hafer KS, Novick AC. Long term results of nephron sparing surgery for localized renal cell carcinoma: 10 year follow up. J Urol. 2000;163(2):442-445.

5. Myrian Garcia Berro, Concha Toribio, Fundación OPTI Nov. 2004

6. Bensalah K, Crépel M, Patard JJ. Tumor Size and Nephronsparing Surgery: Does it Still Matter? European Urology 2008; 53(4):691-693.

7. David S, Finley MD, Shawn Beck MD, William Chu, Geoffrey N Box MD, Duane V, Elspeth M McDougall MD et al. Percutaneous vs. Laparoscopic Cryoablation of small renal masse: Percutaneous is better!.AUA 2008 Abst (951). J Urol 2008; 179 (4)Suppl:327.

8. S. Sumida Short History of Cryosurgery. 14th World Congress of Cryosurgery: 1st International Conference of Cryoinmunology 2007.

9. Pontones Moreno JL, Morera Martínez JF, Vera Donoso CD, Jiménez Cruz JF. Criocirugía en el tratamiento del cancer de Prostata. Actas Urol Esp. 2007;31(3):211-232.

10. Escudero Barrilero A, Arias Fúnez F, Patrón Rodríguez RR, García González R, Cuesta Roca C. Cryobiology and pathologic lesions induced by freezing-thawing processes in prostatic tissue. (Second part) Arch Esp Urol.2004;57(10): 1073-1090. 
11. Gage AA, Baust J. Mechanisms of tissue injury in Criosurgery. Cryobiology.1998;37(3):171-186.

12. Mazur P. Criobiology: The freezing of biological systems Science. 1970;168(934):939-949.

13. Hoffmann NE, Bischof JC. The cryobiology of cryosurgical injury. Urology.2002(2 Suppl 1):40-49.

14. Whittaker DK. Mechanism of tissue destruction following cryosurgery. Ann R Coll Surg Engl. 1984;66(5):313-318.

15. Zook N, Hussman J, Brown R, Russell R, Kucan J, Roth A, Suchy H.. Microcirculatory studies of frosbite injury. Ann Plast surg. 1998;40(3): 246-253.

16. Baust J, Gage AA, MA H, Zhang CM. Minimally invasive cryosurgery-technical advances. Cryobiology 1997;34(4):373-384.

17. Stephenson RA, King DK, Rohr LR. Renal cryoablation in a canine model. Urology 1996;47(5):772-776.

18. Yue-Kun Zou,Ju-Yi Wen, Hua-Song Feng, Yun-You Duan: Apoptosis and the expresión of related genes induced by argohelium-based cryocare system in adenocarcinoma. 14th World Congres of Cryosurgery. 1st International Conference of Cryoimmunology.

19. Uchida $M$, Imaide I,Sugimoto $K$, Uchara $H$, Watanabe $H$ : Percutaneous cryosurgery for renal tumors. Br J Urol.1995; 75(2): 132-137.

20. Preston Sprenkle, Gabriella Mirabile,Gregory W Hruby Courtney K Phillips, Keith Xavier, Martin Gupta et al. The effect of Argon Gas Pressure on Ice ball Size and rate of formation.AUA 2008 Abs (695). J.Urol 2008; 179 (4) Suppl: 243

21. Gill IS, Novick AC, Meraney AM,Chen RN, Hobart MG, Sung GT.H, et al. Laparoscopic renal Cryoablation in 32 patients. Urology 2000; 56(5):748-753.

22. Jaime Landham, Daniel S Lehman, Gregory W Hruby, Courtney K Phillips,Bruce Shingleton. Efficacy and complications of Cryoablation for renal masses; percutaneous vs. laparoscopic ablation.AUA 2007 Abs (1299). J. Urol 2007;177(4)Suppl.

23. Laguna Pes MP, Lagerveld B, Witte LP, Kummerlin I, Wijkstra $\mathrm{H}$, de la Rosette JJ. Crioablación Laparoscópica de las pequeñas masas renales.Actas Urol Esp 2005;29(9):860-868.

24. Cestari A, Guazzoni G, dell'Acqua V, Nava L, Cardone G Balconi G, et al. Laparoscopic cryoablation of Renal masses;intermediate term folow-up. J. Urol 2004;172: 1267-1270.

25. Patricia WT Beemster, Saffire Phoa, Hessel Wijkstra, Jean J de la Rossete, M Pilar Laguna. Follow-Up of renal masses after cryosurgery using computerized tomography enhancement patterns and cryolesion size.AUA Abs (1243). J. Urol 2007; 177 (4) Suppl.

26. Stein AJ, Mayes JM, Mouraviev V, Caen VH, Nelson RC, Polascik TJ. Persistent contrast enhancement several months after laparoscopic cryoablation of the small renal mass may no indicate remaining tumor. J Endourol 22(11): 2433-2440.

27. Young Hwii Ko,Seok Ho Kang, Je Hyun Bae, Du Geon Moon, Hong Seok Park, Jun Geon et al. Tumor-Specific immunity induced by cryoablation in a murine renal cell carcinoma rechallenge model.AUA 2008 Abs (111). J. Urol 2008;170(4) Suppl.:39.

28. Mohamed Ismail, Richard Morgan, Jhon Davies and Hardev Pandha. Inmunoregulatory effects of Cryo-treated prostate cancer cells on human dendritic cells using a novel in vitro cryotherapy model. AUA 2008 Abs (136). J. Urol 2008;179 (4)Suppl.:48

29. Richard J. Ablin. Cryoinmunology: Wanderinging in the Desert for 40 years. 14th World Congress in Cryosurgery: 1st International Conference of Cryoinmunology 2007.

30. Valèrie Forest. Cryoscience. 14th World Congress in Cryosurgery: 1st International Conference of Cryoinmunology 2007: 146.
31. Benjamin A, Tjoa,Ph.D. Antigen-Presenting cells, Limphocytes, and Citokines. Their potencial roles in Cryo-Inmunotherapy. 14th World Congress in Cryosurgery: 1st International Conference of Cryoinmunology 2007.

32. Michael M. Sabel. Evidence oy Inmune response to Cryotherapy. 14th World Congress in Cryosurgery: 1st International Conference of Cryoinmunology 2007.

33. Lea Eisenbach et al. Cryoinmunotherapy: Mechanism and preclinical Studies. 14th World Congress in Cryosurgery: 1st International Conference of Cryoinmunology 2007:147.

34. William A. Cavanagh. Biology of Cryoabaltion. Potential mechanisms Toward a Cryoinmuno-effect. 14th World Congress in Cryosurgery: 1st International Conference of Cryoinmunology 2007:147.

35. Yutaka Kawakami. Inmunoterapy by intramural administration of dendrtic cells following cryoablatio tumor treatment.14th World Congress in Cryosurgery: 1st International Conference of Cryoinmunology 2007.

36. Richard $\mathrm{J}$ Ablin Observations of Systemic Cryo-Immunity and Rationale for Cryoimmunotherapy A historical perspective. 14th World Congress in Cryosurgery: 1st International Conference of Cryoinmunology 2007:148.

37. Adema GJ et al. Synergy between in situ tumor destruction and immune stimulation results in highly effectve dendrtic cell bacines in vivo. 14th World Congress in Cryosurgery: 1st International Conference of Cryoinmunology 2007.

38. Carvalhal EF, Gill IS,Meraney AM,Desai MM Schweizer DK, Sung GT. Laparoscopic renal cryoablation: Impact on renal function and blood pressure. Urology 2001; 58(3):357-361.

39. Monish Aron, Kamuzi Kamoi,Geroges-Pascal Haber, Mihir M Desai, David Canes, Jihad H Kaouk, Indebir S Gill. Laparoscopic renal cryoablation: Long-term oncologic outcomes with minimun 5-year follow-up. AUA 2008 Abs (596). J. Urol 2008;179 (4)Suppl.:209.

40. Rukstalis DB, Khorsandi M, Garcia FU, Hoening DM, Cohen JK. Clinical experience with open renal cryoablation. Urology 2001Jan;57(1):34-39.

41. Johnson DB, Solomon SB, Su LM, Matsumoto ED, Kavoussi LR, Nakada SY, Moon TD, Shingleton WB, Cadeddu JA.. Defining the complications of cryoablation and radiofrecuency ablation of small renal tumors; a multiinstitutional review. J. Urol 2004; 172(3): 874-877.

42. Justin Sausville, James Borin, Michael W Phelan. Initial experience in renal cryosurgery for large renal lesions. AUA 2008 Abs (831). J.Urol 2008; 179 (4)Suppl.:288.

43. Clarke DM, Robilotto AT,Rhee E, Van Buskirk RG, Baust JG, Gage AA, Baust JM. Cryoablation of renal cancer: variables involved in freezing-induced cell death. Technol Cancer Res Treat. 2007;6(2):69-79.

44. Stein RJ, Kaouk JH. Renal cryotherapy: A detailed review including a 5-year follow-up. BJU Int. 2007;99(5 Pt B):12651270 .

45. Hegarty NJ, Kaouk JH, Eric Remer, CH.M. O`Malley. Indebir S. Gill, Novick AC.Laparoscopic renal cryoablation: oncological outcomes at 5 years. J Endourol; 2006;20(10):753-760.

46. Davol PE, Fulmer BR, Rukstalis DB. Long-term results of cryoablation for renal cancer and complex renal masses. Urology 2006;68(Suppl.):2-6.

47. Weld KJ, Figenshau RS, Venkatesm R, Bhayani SB, Ames CA, Clayman RV, Landman J. Laparoscopic Cryoablation for small renal masses:Three-year follow-up. Urology 2007; 69:448-451.

48. Fotiadis NI, Sabharwal T, Morales JP, Hodgson DJ, O'Brien TS, Adam A. Combined percutaneous radiofrequency ablation and etanol injection of renal tumors:Midterm results. Eur Urol. 2007 Sep;52(3):777-784. 
49. Langenkamp S, Abusalim N, Huenerbein R, Khun FP, Albers P. Percutaneous Radiofrequency ablation of renal TumoursEmbolization before RFA.Eur Urol Supplements, 2008:(7):309 309.

50. Svatek RS, Sims R, Anderson JK, Abdel-Aziz K, Cadeddu JA Magnetic Resonance Imaging Characteristics of Renal Tumors after Radiofrequency ablation. Urology 2006;67(3):508-512.

51. Mayo-Smith WW, Dupuy DE,Parikh PM, Perullo JA, CronanJJ. Imaging-guided percutaneous radiofrequency abaltion of solid renal masses techniquea and outcomes of 38 treatment sessions in 32 consecutives patients AJR Am J Roentgenol. 2003; 180(6): 1503-1508

52. Kassoouf W, Aprikian Ag, Laplante M, Tanguay S. Natural his tory of renal masses followed expectantly. J. Urol 2004 Jan; 171 (1):111-113.

53. Chow WH, Devessa SS, Warren Jl. Rising incidence of renal cancer in the Unitd States 1999;281(17):1628-1631.

54. Katz DL, Zheng T, Holford TR, Flannery J. . Time trends in the incidence of renal cell carcinoma analysis of Conneticut tumor registry data 1935-1989.Int J Cancer. 1994;58(1):57-63.

55. Sowery RD, Siemens DR. Growth characteristics of renal cortical tumors in patients managed by watchful waiting. Can J Urol 2004;11(5):2407-2410.

56. Bosniak MA. Observation of small incidentally detected renal masses. Semin. Urol Oncol.1995;13(4):267-272.

57. Wehle MJ, Thiel DD,Petrou SP, Young Pr, Frank I, Karsteadt N Conservative management of incidental contrst-enchancing renal masses as safe to invasive therapy. Urology, 2004; 64(1):49-52.

58. Lamb GW, Bromwich EJ,Vasey P, Aitchison M. Management of renal masses in patients medically unsuitable for nephrectomy-natural history, complications, and outcome. Urology, 2004;64(5):909-913

59. Chawla SN, Crispen PL, Hanlon AL. The Natural history observed enhancing renal masses meta-analysis and review of the world literature. J.Urol 2006;175.425-531.

60. Curry NS, Biassada NK. Radiologic evaluation of small and indeterminant renal masses. Urol Clin North Am 1997;24:493505.

61. Wong JA, Rendon RA. Can Urol Assoc J.2007;(2): 120-122.

62. Crispen PL, Viterbo R, Fox EB, Greenberg RE, Chen DY, Uzzo RG. Delayed intervention of sporadic renal masses undergoing active surveillance. Cancer, 2008;112(5):1051-1057.
63. Kouba E, Simth A, McRackan D, Wallen EM, Pruthi RS. Watchful waiting for solid renal masses:insight into the natural history and results of delayed intervention. J Urol, 2007;177 (2):466-670.

64. Volpe A, Panzarella T,Rendón RA, Haider MA, Kondylis FI, Jewett MA. The natural history of incidentally detected small renal masses. Cancer, 2004 Feb 15;100(4):738-745.

65. Rendon RA, Jewett MA. Expectant management for the treatment of small renal masses. Urol Oncol. 2006;24(1):62-67.

66. Volpe A, Jewet MA. The natural history of small renal masses. Nat Clin Pract Urol, 2005 Aug;2(8):384-390.

67. Mattar K, Jewett MA. Watchful waiting for small renal masses. Curr Urol rep. 2008;9(1):22-25.

68. Matsuzaki M, Kawamo Y, Morikawa H, Siga Y, Murata H, Komatsu H. Conservative management of samll renal tumors. Hinoyika Kiyo. 2007;53(4): 207-211.

69. Kunkle DA, Egleston BL, Uzzo RG. Excise, Ablate or Observe: The small renal mass dilema-a meta-analysis, and review. J Urol. 2008 (179): 1227-1234.

70. Volpe A. The role of surveillance in the management of small renal masses, ScientificWorldJournal. 2007; 30(7:)860-868.

71. Peter Whelan. Non-Surgical ablative treatments for small renal tumors less than $4 \mathrm{~cm}$. 2006; 533-536. [European Urology Suplement.

72. Kunkle DA, Egleston BL, Uzzo RG. Excise, ablate or observe: the small renal mass dilemma-a meta-analysis and review. J. Urol, 2008;179(4):1227-1233.

73. Layron Long,Sangtae Park. Difference in patterns of care, reablation and nephrectomy rates after needle ablative therapy for renal masses by Urologists and Radiologists. J. Urol 2007; 177 Suppl.(4):(\#1790).

Correspondencia autor: Dr. José I. Iriarte Soldevilla Servicio de Urología. Hospital de Basurto.

Avda. Montevideo, 18 - 48013 Bilbao - Vizcaya

Tel.: 944006160

E-mail autor: joseignacio.iriartesoldevilla@osakidetza.net

Información artículo: Original

Trabajo recibido: marzo 2009

Trabajo aceptado: abril 2009 